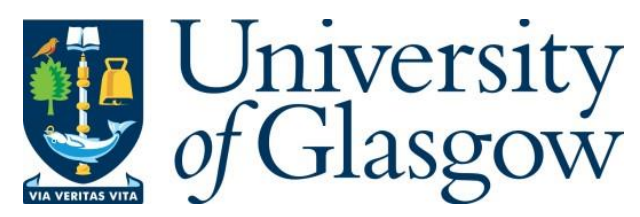

Mathur, N., Summan, R., Dobie, G., West, G. and Marshall, S. (2017) Error Model of Misalignment Error in a Radial 3D Scanner. In: IEEE Sensors 2017, Glasgow, UK, 29 Oct - 01 Nov 2017, ISBN 9781509010127.

There may be differences between this version and the published version. You are advised to consult the publisher's version if you wish to cite from it.

http://eprints.gla.ac.uk/197283/

Deposited on: 5 March 2020

Enlighten - Research publications by members of the University of Glasgow http://eprints.gla.ac.uk 


\title{
Error Model of Misalignment Error in a Radial 3D Scanner
}

\author{
Neha Mathur, Rahul Summan, Gordon Dobie, Graeme West, Stephen Marshall \\ Department of Electronic and Electrical Engineering \\ University of Strathclyde \\ Glasgow, United Kingdom
}

\begin{abstract}
A radial 3D, structured light scanner, was developed from a laser projector and a wide field of view machine vision camera to inspect two - four inch diameter pipers, primarily in the nuclear industry. For identifying the nature and the spatial extent of defective regions, the system constructs a surface point cloud. A dominant source of error in the system is caused by manufacturing tolerances which leads to misalignment between the laser projector and the camera. This causes a triangulation error, reducing the accuracy of the result. In this paper, the error model of the misalignment of the laser and image plane. For a given target distance, we derive an almost linear relationship between angular error in degrees and the error in reported radius (distance from the probe to the surface) in $\mathbf{~ m m}$ and found that for the target $0.1 \mathrm{~mm}$ accuracy on a 4 inch pipe, the misalignment needs to be controlled to less than 0.05 degrees. Future work will consider a post manufacturing calibration routine to compensate for this misalignment.
\end{abstract}

Keywords-Laser Profiler; Structure from Motion; Systematic Error; Visual Inspection

\section{INTRODUCTION}

Remote internal visual inspection of the pipework in the nuclear industry is a periodic activity done to ensure the safe operation of the plant. Currently such inspection is carried out by the operator by monitoring the live camera view of the surface. This can be challenging as with distance, observing the surface defects can be difficult. A probe system consisting of a laser profiler is developed. The geometry of the surface can be inferred by tracking the camera motion and constructing a surface point cloud. For the visualisation of the pipe, the generated point cloud transformed into a triangular mesh using a meshing algorithm. To obtain a photorealistic, geometrically accurate surface model, the image data is mapped onto to the mesh through a texturing procedure.

However, the accuracy of the reconstruction is affected by a number of errors which can be broadly classified as systematic errors and sampling errors respectively. It is important to understand these errors, in order to fully understand the accuracy of the measurement system. The focus of this paper is to describe an error model of the systematic error introduced by the misalignment in the laser profiler and then detail the accuracy evaluation.

\section{BACKGROUND}

The probe consists of a 2MP machine vision camera coupled with a fisheye lens. Thus, in a single image, a $360^{\circ}$ cross sectional view of the surface is captured where the lateral extent

This work was funded by Innovate UK in the "Mosaicing for Automated Pipe Scanning" project. is a function of pipe diameter. This leads to quicker inspections at the cost of increased signal processing [1] - [4]. The current hardware prototype is shown in Figure 1 where each subsystem is highlighted.

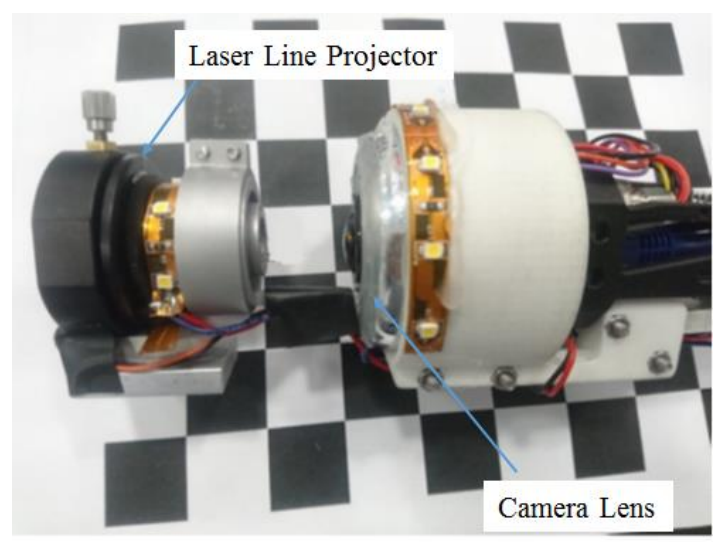

Fig. 1. The camera and laser system.

In order to profile the pipe interior, the probe system is placed inside the pipe. Here a circular pattern is projected by the probe onto the inner pipe wall. As the probe is driven through the pipe, it captures images. These images contain information which are then utilized to measure the shape of the pipe and to detect any surface defects. The geometry of the laser and camera configuration is shown in Figure 2.

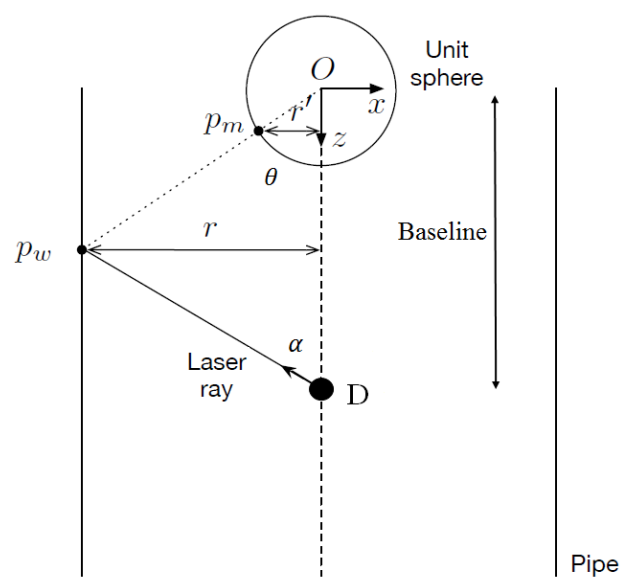

Fig. 2. The geometry of the laser and camera. 
This arrangement contains two geometric parameters, the baseline OD and the opening angle $\alpha$, which are needed for triangulation - these can be obtained through camera calibration prior to operation by the camera model described by Scaramuzza et al [5]. In our arrangement, the laser is emitted orthogonally to the optical axis of the camera corresponding to an angle $\alpha=180$ degrees. With the knowledge of the baseline length $l$ and the direction vector corresponding to each laser pixel, triangulation can be employed to estimate the $3 \mathrm{D}$ position of each these pixels.

A comprehensive list of errors sources for our system is shown in Table I. In this work, the systematic error due to the misalignment between the laser plane and the camera plane is investigated.

TABLE I. SOURCES OF ERROR IN THE LASER PROFILER

\begin{tabular}{|c|c|}
\hline \multicolumn{2}{|r|}{ Errors } \\
\hline Systematic Error & $\begin{array}{l}\text { 1. Misalignment - manufacturing } \\
\text { tolerances, optical axis and laser } \\
\text { misaligned w.r.t. pipe } \\
\text { 2. Opening angle } \alpha \\
\text { 3. Baseline length } l \\
\text { 4. Focal length of the camera } f_{c} \\
\text { 5. Calibration of the camera }\end{array}$ \\
\hline Sampling (Random) Error & $\begin{array}{l}\text { 1. Variable laser line width } \\
\text { 2. General image noise }\end{array}$ \\
\hline
\end{tabular}

\section{SYSTEMATIC ERROR - MISALIGNMENT}

Systematic errors [7] are due to the geometric errors in the system due to manufacturing tolerances. Unlike sampling errors, they can't be averaged out and instead are typically addressed through calibration.

It is assumed that the laser plane is parallel to the image plane. Error in laser angular alignment propagates through to the measurement error of the depth of a laser point. This leads to error in triangulation which is a function of the laser angular misalignment. Also, alignment error can occur if the optical axis and the laser are not aligned with the pipe axis leading to inaccuracy in feature extraction [6]. The alignment error needs to be examined in case of a pure translation or pure rotation. As in [7], [8] the translational alignment error is corrected by reconstructing the projected laser curve and estimating the orientation of the central pipe axis with respect to the camera reference frame. The next step is transforming this to a coordinate frame whose $z$-axis is parallel with that of the pipe reference [9]-[13]. But for accurate image reconstruction, the rotational alignment error needs to be analyzed as well.

To evaluate this, consider Figure 3 which represents the misalignment of the laser w.r.t the optical axis. If the laser and camera system are misaligned by a distance $x$, corresponding to misalignment angle $\varnothing$, then the location of the world point is $p_{w}$, and $\theta^{\prime}$ is the angle between the vector $p_{m}$ (extended from the origin) and the $z$-axis of the probe. Mathematically, the error when the laser is misaligned from optical axis can be derived as

$$
\text { Error }=r\left[\frac{\tan \theta \tan \emptyset}{1+\tan \theta \tan \varnothing}\right]
$$

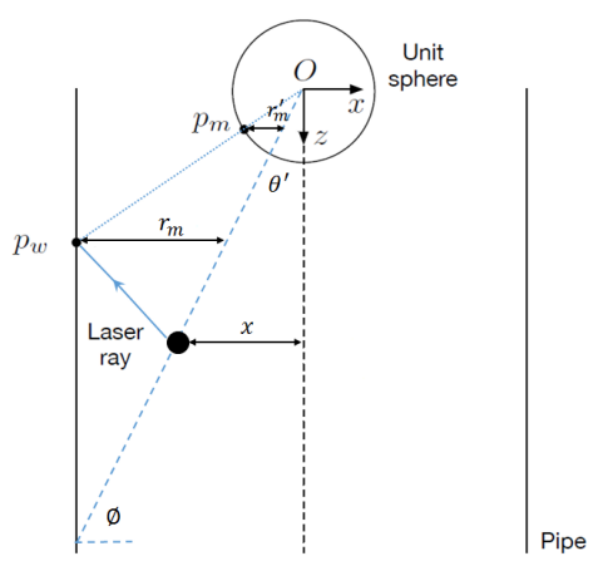

Fig. 3. Illustration of the geometry of the laser and camera when misaligned.

\section{ERROR MODEL}

In the ideal scenario (as in Figure 2) the probe is positioned exactly in the middle of the pipe, then the laser would project a circle of radius $r \in R_{+}$say, onto the surface of the pipe. The same circle would be reflected on the surface of the unit sphere, however, with a proportionally smaller radius, $r^{\prime} \in R_{+}$. Prior to the operation, the camera is calibrated to determine the mapping from a $3 \mathrm{D}$ scene point to a $2 \mathrm{D}$ image point with sub-pixel accuracy. The camera calibration procedure uses the so-called sphere of equivalence model to model the mirror of the camera system. This can be given by Thales' theorem as

$$
\frac{\left\|p_{w}\right\|}{r}=\frac{\left\|p_{m}\right\|}{r^{\prime}}
$$

The radius of the circle projected onto the surface of the unit sphere, $r^{\prime}$, on the other hand, can be found numerically by fitting a circle to the set of points reprojected from the captured image onto the unit sphere using the OCamCalib toolbox developed by Scaramuzza et al [5].

However, when the laser is misaligned (as in Figure 3) then $r_{m}$ is not equal to the inner pipe diameter and hence the radius of the projected circle on the unit sphere $r_{m}^{\prime}$ would be incorrect leading to triangulation error. In order to investigate the effect of the misalignment of the laser (in terms of the tilt angle $\emptyset$ ) on triangulation, an error model GUI was designed in Matlab. This tool enables to simulate the effect of laser-camera misalignment by using a real camera model from the probe. The error is calculated as the Euclidean distance between the individual points on the actual laser ring and the circle obtained by triangulating the captured image onto the unit sphere. The Euclidean distance between points $p$ and $q$ is given by 


$$
\|q-p\|=\sqrt{\|p\|^{2}+\|q\|^{2}-2 p \cdot q}
$$

\section{RESULTS}

Figure 4 depicts the simulated triangulation error for a pipe of diameter 4 inch when the laser is misaligned with an angle 0.6 degrees.
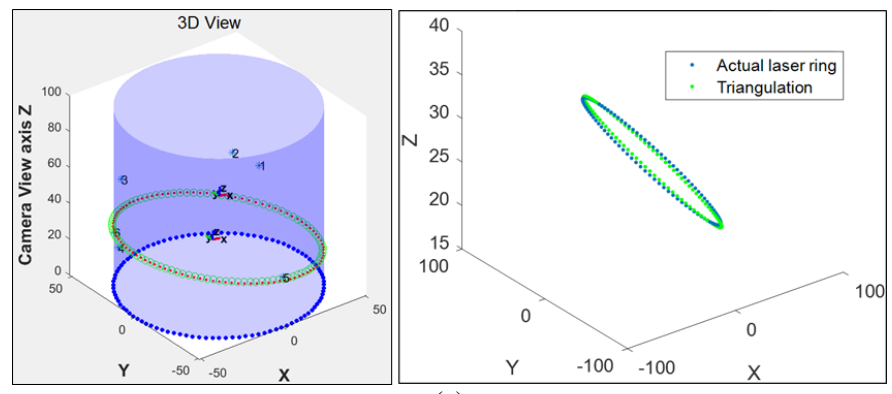

(a)

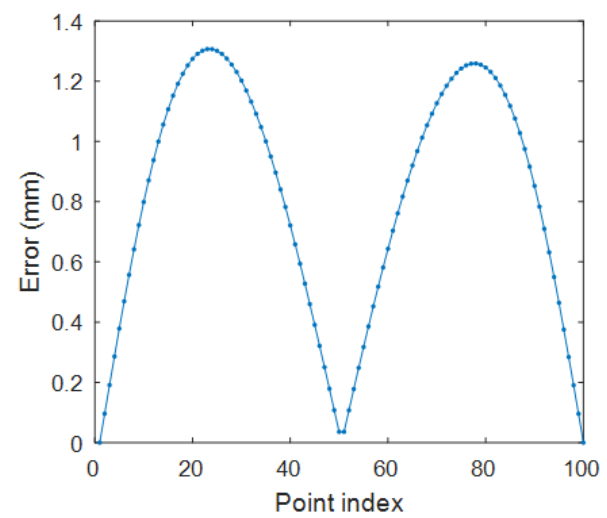

(b)

Fig.4. Simulated laser-camera misalignment for a 4 inch pipe with tilt angle 0.6 degrees: (a) Actual laser ring along with the triangulated in 3D view (b) Euclidean distance for each of the laser points.

Figure 5 shows the maximum error as a function of angular misalignment for three different pipes diameters: 2,3 and 4 inch which indicates that the angular misalignment error increases with the increase in the pipe diameter.

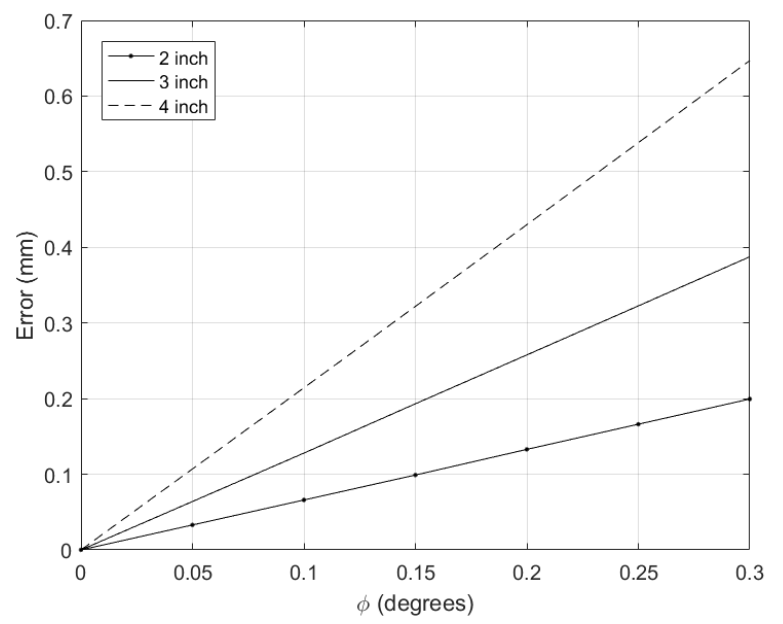

Fig.5. Maximum triangulation error as a function of laser misalignment for pipes of different diameters.

\section{FUTURE WORK}

Future work would seek to experimentally validate the triangulation error due to misalignment of the laser and the camera plane. This would involve aligning the camera system within a pipe of known dimensions and known features. From these features, the camera's position can be inferred through the Perspective-n-Point algorithm. Once the error is validated, the team will consider a calibration routine to compensate for this source of error.

\section{ACKNOWLEDGMENT}

This work was funded by Innovate UK in the "Mosaicing for Automated Pipe Scanning" project (Innovate UK -102067). The authors would like to thank the project partners: National Nuclear Laboratory, Wideblue, Inspectahire and Sellafield Ltd.

\section{REFERENCES}

[1] J. Kannala, S. S. Brandt, and J. Heikkila, "Measuring and Modelling Sewer Pipes from Video," Machine Vision and Applications, vol. 19, no. 2, pp. 73-83, March 2008.

[2] P. Hansen, H. Alismail, P. Rander, and B. Browning, "Pipe Mapping with Monocular Fisheye Imagery," Proc. of IEEE/RSJ International Conference on Intelligent Robots and Systems (IROS), pp. 5180-5185, Novemeber 2013.

[3] K. Matsui, Y. Atsushi, and K. Toru, "3-D Shape Measurement of Pipe by Range Finder Constructed with Omni-directional Laser and Omnidirectional Camera," IEEE International Conference on Robotics and Automation (ICRA), pp. 2537 - 2542, May 2010.

[4] P. Murray, G. West, S. Marshall, S. McArthur, "Automated image stitching for enhanced visual inspections of nuclear power stations," $10^{\text {th }}$ International Conference on Condition Monitoring and Machine Failure Prevention Technologies, June 2013.

[5] D. Scaramuzza, A. Martinelli, and R. Siegwart, "A Flexible Technique for Accurate Omnidirectional Camera Calibration and Structure from Motion," $4^{\text {th }}$ IEEE International Conference on Computer Vision Systems (ICVS'06), January 2006.

[6] E.C. Dertien, "Design of an inspection robot for small diameter gas distribution mains." Doctoral dissertation, University of Twente, 2014.

[7] E. Drost, "Measurement System for pipe profiling." MSc Report, University of Twente, 2009.

[8] M. Reiling, "Implementation of a Monocular Structured Light Vision System for Pipe Inspection Robot PIRATE." Master's thesis, university of Twente, 2014.

[9] A.D. Tezerjani, M. Mehrandezh, R. Paranjape, "4-DOF pose estimation of a pipe crawling robot using a Collimated Laser, a conic mirror, and a fish-eye camera," IEEE Southwest Symposium on Image Analysis and Interpretation (SSIAI), pp. 45-48, 2014.

[10] P.V. Unnikrishnan, B. Thornton, T. Ura, Y. Nose, "A conical laser lightsectioning method for navigation of autonomous underwater vehicles for internal inspection of pipelines," IEEE OCEANS 2009-EUROPE, pp. 19, May 2009.

[11] P. Buschinelli, T. Pinto, F. Silva, J. Santos, A. Albertazzi, "Laser Triangulation Profilometer for Inner Surface Inspection of 100 millimeters (4") Nominal Diameter," Journal of Physics: Conference Series, IOP Publishing, vol. 648, no.1, 2015.

[12] A. Natraj, C. Demonceaux, P. Vasseur, P. Sturm, " Vision based attitude and altitude estimation for UAVs in dark environments," IEEE International Conference on Intelligent Robots and Systems (IROS), pp. 4006-4011, October 2012.

[13] A. D. Tezerjani, "High Resolution Visual Pipe Characterization System Using an Omnidirectional Camera." Faculty of Graduate Studies and Research, University of Regina, 2015. 